\title{
Arbeits(un)fähigkeit bei psychischen Erkrankungen
}

\author{
Hintergründe, sozialpolitische Dimensionen, empirische \\ Daten und Perspektiven
}

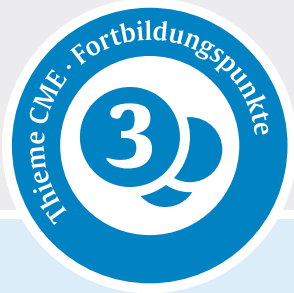

Andreas Hillert ${ }^{1}$, Tabea Bauman ${ }^{1}$, Adrian Meule ${ }^{1,3}$, Ute Heinrich ${ }^{2}$

1 Schön Klinik Roseneck, Prien am Chiemsee

2 AUDI AG, Ingolstadt

3 Klinik für Psychiatrie und Psychotherapie, LMU Klinikum, München

\section{ZUSAMMENFASSUNG}

In einer Expertenbefragung benannten Ärzte und Psychologen erhebliche Dilemmata hinsichtlich der Feststellung von Arbeitsunfähigkeit angesichts psychiatrischer/psychosomatischer Patienten. Routinedaten einer psychosomatischen Fachklinik zeigen eine signifikante Reduktion z. B. der depressiven Symptomatik von Patienten im Therapieverlauf, ohne dass dies unmittelbar und in jedem Fall mit einer Zunahme der Arbeitsfähigkeit bei Entlassung korreliert. Limitiert wird dieser Befund durch das Fehlen von Daten zum weiteren poststationären Verlauf. Die bezüglich der Feststellung von Arbeitsunfähigkeit intervenierenden Aspekte werden eingehend diskutiert. Von den befragten Experten und Klinikern wird u. a. eingeräumt, dass die Selbsteinschätzung der Patienten erheblichen Einfluss die die letztlich vollzogene Bewertung hat. Ausgehend hiervon werden die offiziellen Richtlinien zum Thema Arbeitsunfähigkeit referiert. Wissenschaftliche Studien zur Reliabilität von Krankschreibungen gibt es nicht, was u. a. die Aussagekraft von Präsentismus-Studien relativiert. Konzepte, die der immensen Tragweite für alle Beteiligten und der entsprechenden Komplexität des Themas angemessen sind, werden skizziert. Adäquate Kenntnisse der Arbeitsbedingungen ihrer Patienten, Wissen hinsichtlich der Ambiguität von Krankschreibungen (pathogene und salutogenetische Aspekten der Arbeit) und eine (Selbst-)Reflexion bezüglich des Themas erscheinen unabdingbar. Zumal angesichts von offenkundigen Arbeitskonflikten und Überlastungskonstellationen der Patienten wäre es angemessen, anstatt längerfristiger Krankschreibungen, Überlastungsanzeigen aus medizinisch/psychotherapeutischen Gründen zu machen und eine systemisch-interdisziplinäre Lösung zum Nutzen aller Beteiligter anzustreben.

\section{Einführung und Hintergrund}

„Wer krank ist, der kann und darf nicht arbeiten - und ob man krankheitsbedingt nicht arbeiten kann, muss letztlich jeder selber für sich entscheiden. “ Dieses - professionell gesehen schwierige - Postulat gehört quasi zum Allgemeinwissen. Im Internet finden sich klare diesbezügliche Instruktionen:

„Wenn Sie aufgrund einer Erkrankung nicht arbeiten können, müssen Sie einen Arzt aufsuchen, der Ihnen Ihre Arbeitsunfähigkeit schriftlich bescheinigt. Die meisten $\mathrm{Ar}$ beitnehmer fallen dann oftmals einige Tage, in seltenen Fällen sogar mehrere Wochen oder Monate aus ... Die Verweigerung einer Arbeitsunfähigkeitsbescheinigung kann den Arzt aufgrund eines mitunter langjährigen Vertrauensverhältnisses in eine schwierige Situation bringen ..." (www.anwalt.org/arbeitsunfaehigkeit).

Dass Stress, zumal in der Arbeit, potenziell krank und Erholung, im Sinne von „nicht arbeiten müssen“, gesund macht, dürfte parallel dazu von der Bevölkerungsmehrheit als zutreffend beurteilt werden [1]. Diese populären Ansichten zu "Gesundheit und Krankheit" können keine naturwissenschaftliche Richtigkeit für sich beanspruchen. Sie sind das Ergebnis historischer Entwicklungen, die in den westlichen (Post-)Industrienationen durch Wohlstand und Individualismus gekennzeichnet sind [2, 3]. Selbstverständlich kann ein schwer depressiver oder akut psycho- tischer Mensch keiner Erwerbsarbeit nachgehen. Wenn er am Arbeitsplatz erscheinen würde, wäre er ein Risiko für sich und andere. Jenseits solcher Extrem-Kategorien wird es schwieriger: Wo hört psychische Gesundheit auf, wo fangen Erkrankungen an? Wie wird davon ausgehend der alltägliche Akt „Krankschreibung“ gehandhabt? Diese Fragen sind nicht zuletzt von erheblicher sozialpolitischer Tragweite [4]. Krankenkassenstatistiken belegen eine seit Jahren zunehmende Häufigkeit von Krankschreibungen aufgrund psychischer Diagnosen [5], ohne dass psychische Erkrankungen in der erwachsenen Bevölkerung insgesamt zugenommen haben [6, 7]. Neben erhöhter Offenheit von Ärzten und Gesellschaft (Entstigmatisierung) psychischen Erkrankungen gegenüber, wird dies als Folge zunehmenden Druckes in der Arbeitswelt erlebt. Jede weitergehende Interpretation setzt voraus, die Kriterien zu kennen, nach denen im Alltag „arbeitsunfähig“ - also AU - geschrieben wird. Parallel zum Anspruch, auf Basis objektiver, reliabler und letztlich valider Befunde [8], jedem Patienten die angemessene Diagnose und die richtige Behandlung zukommen zu lassen, sollte auch an Krankschreibungen eine entsprechend hohe, wissenschaftlichen Kriterien entsprechende Messlatte angelegt werden.

Das Phänomen „Krankschreibung“ wurde theoretisch-konzeptuell diskutiert $[9,10]$, empirische Daten zu den im Alltag realiter angelegten Kriterien fehlen. Sind Krankschrei- 
bungen das Ergebnis rationaler Abwägungen von „objektiv erhobenen Einschränkungen“ relativ zu „objektiven Anforderungen am aktuellen bzw. letzten Arbeitsplatz“ eines Menschen (unter Berücksichtigung u.a. der Abwendung relevanter gesundheitlicher Risiken)? Falls nein, welche anderen Aspekte haben Einfluss auf „Krankschreibungen“? Ziel und Inhalt dieses Beitrages sind es, hierzu 2 - allererste empirische - Erhebungen vorzustellen:

- eine systematische Dokumentation von Krankschreibungen psychosomatischer Patienten vor und nach einem Klinikaufenthalt und zum anderen

- eine Befragung von Ärzten und psychologischen Psychotherapeuten zu eigenen Erfahrungen und ihrem Umgang mit dem Thema Arbeitsunfähigkeit.

\section{Arbeits(un)fähigkeit vor und nach stationärer psychosomatischer Akutbehandlung}

\section{Material und Methoden}

Es wurden die Daten aller unter der Hauptdiagnose Depression (F32 und F33 nach ICD-10) zwischen 2015 und 2020 in einer psychosomatischen Fachklinik (Schön Klinik Roseneck, Prien) behandelten erwachsenen Patienten ausgewertet (soweit die Information arbeitsfähig bei Aufnahme und Entlassung dokumentiert wurde, $n=3053$ ). Die depressive Symptomatik wurde durch Selbstbeurteilung anhand des Patient Health Questionnaire (PHQ-9) [11-13] bei Aufnahme und Entlassung erfasst. Die Verbesserung des allgemeinen Funktionsniveaus während des Aufenthalts wurde durch Fremdbeurteilung der Bezugstherapeuten anhand der Clinical Global Impression-Improvement Scale bei Entlassung erfasst [14]. Die Veränderung der depressiven Symptomatik in Abhängigkeit der Arbeitsfähigkeit wurde mithilfe einer Messwiederholungs-Varianzanalyse getestet mit dem Innersubjektfaktor Zeitpunkt (Aufnahme vs. Entlassung) und den Zwischensubjektfaktoren Arbeitsfähigkeit vor Aufnahme (ja vs. nein) und Arbeitsfähigkeit bei Entlassung (ja vs. nein). Die Verbesserung des allgemeinen Funktionsniveaus in Abhängigkeit der Arbeitsfähigkeit wurde mithilfe einer univariaten Varianzanalyse getestet mit den Zwischensubjektfaktoren Arbeitsfähigkeit vor Aufnahme (ja vs. nein) und Arbeitsfähigkeit bei Entlassung (ja vs. nein). Zur Interpretation von Interaktionseffekten wurden t-Tests für unabhängige Stichproben verwendet.

\section{Ergebnisse}

Von den einbezogenen Patienten (Alter $M=44,4$ Jahre, $\mathrm{SD}=13,8$, Range: $18-78)$ waren 1724 weiblich (56,5\%) und 1329 männlich (43,5\%). Fast die Hälfte der Patienten hatte mindestens eine komorbide psychische Erkrankung ( $n=1491$, 48,8\%; M = 0,72 komorbide psychische Erkrankungen, SD =0,91, Range: 0-6). Die mittlere Verweildauer betrug 49,6 Tage (SD = 21,2, Range: 1-231). Von diesen Patienten waren $n=761$ arbeitsfähig bei Aufnahme und
Entlassung, $n=233$ arbeitsunfähig bei Aufnahme, arbeitsfähig bei Entlassung, n= 556 arbeitsfähig bei Aufnahme, arbeitsunfähig bei Entlassung, und $n=1503$ arbeitsunfähig bei Aufnahme und Entlassung. Das heißt auf deskriptiver Ebene wurden durch den Aufenthalt, einhergehend insgesamt mit einer deutlichen Verbesserung der depressiven Symptomatik, 233 zunächst arbeitsunfähige Patienten arbeitsfähig und 556 zunächst arbeitsfähige Patienten arbeitsunfähig. Bei den meisten Patienten $(n=2264)$ hatte die mehrwöchige Behandlung demnach keinen Einfluss auf deren $\mathrm{AU}$.

Aufgrund fehlender Werte basieren die Analysen mit dem PHQ-9 auf $n=2418$ Patienten. Es zeigte sich eine signifikante 3-Fachinteraktion Zeitpunkt $\times$ Arbeitsfähigkeit vor Aufnahme $\times$ Arbeitsfähigkeit bei Entlassung $(F(1,2141)=7,84$, $\left.p=0,005, \eta^{2} p=0,003\right)$. Wie aus $>$ Abb. 1 ersichtlich wird, hatten Patienten, die AU aufgenommen wurden, aber arbeitsfähig entlassen wurden, die stärkste Reduktion depressiver Symptomatik, die signifikant höher war als in den 3 anderen Gruppen (alle p <0,023). Während diese Gruppe bei Aufnahme noch eine gleich hoch ausgeprägte Symptomatik aufwies wie Patienten, die bei Aufnahme arbeitsfähig, aber bei Entlassung AU waren ( $t(720)=0,22, p=0,826$ ), hatten sie bei Entlassung signifikant niedrigere Werte $(t(661)=2,24, p=0,025)$. Gleichzeitig hatte diese Gruppe bei Aufnahme noch eine signifikant stärker ausgeprägte depressive Symptomatik als Patienten, die sowohl bei Aufnahme und Entlassung arbeitsfähig waren $(t(904)=2,40$, $p=0,017)$, aber unterschied sich von dieser nicht mehr bei Entlassung $(t(863)=0,92, p=0,359)$. Zudem zeigten Patienten, die sowohl bei Aufnahme und Entlassung arbeitsfähig waren, eine signifikant stärkere Reduktion depressiver Symptomatik als Patienten, die sowohl bei Aufnahme als auch Entlassung AU waren $(t(1786)=1,98, p=0,047)$. Die Veränderung der depressiven Symptomatik bei Patienten, die bei Aufnahme arbeitsfähig waren, aber bei Entlassung $\mathrm{AU}$, unterschied sich nicht signifikant zu Patienten, die sowohl bei Aufnahme als auch Entlassung arbeitsfähig bzw. AU waren (beide $\mathrm{p}>0,120$; $>$ Abb. 1).

Aufgrund fehlender Werte basieren die Analysen mit der Clinical Global Impression-Improvement Scale auf $n=2949$ Patienten. Es zeigte sich eine signifikante Interaktion Arbeitsfähigkeit vor Aufnahme $\times$ Arbeitsfähigkeit bei Entlassung $\left(F(1,2945)=7,33, p=0,007, \eta^{2} p=0,002\right)$. Wie aus $\triangleright$ Abb. 2 ersichtlich wird, zeigten - entsprechend den Ergebnissen des PHQ-9-Patienten, die AU aufgenommen wurden, aber arbeitsfähig entlassen wurden, die stärksten Verbesserungen während des stationären Aufenthalts, wobei sich diese nicht signifikant von den Verbesserungen der Patienten unterschieden, die sowohl bei Aufnahme als auch Entlassung arbeitsfähig waren $(t(964)=0,77$, $\mathrm{p}=0,442)$. Alle anderen Gruppen unterschieden sich signifikant voneinander (alle $p<0,005$ ), wobei Patienten, die $\mathrm{AU}$ aufgenommen und entlassen wurden, die geringsten Verbesserungen zeigten ( $\triangleright$ Abb. 2 ). 


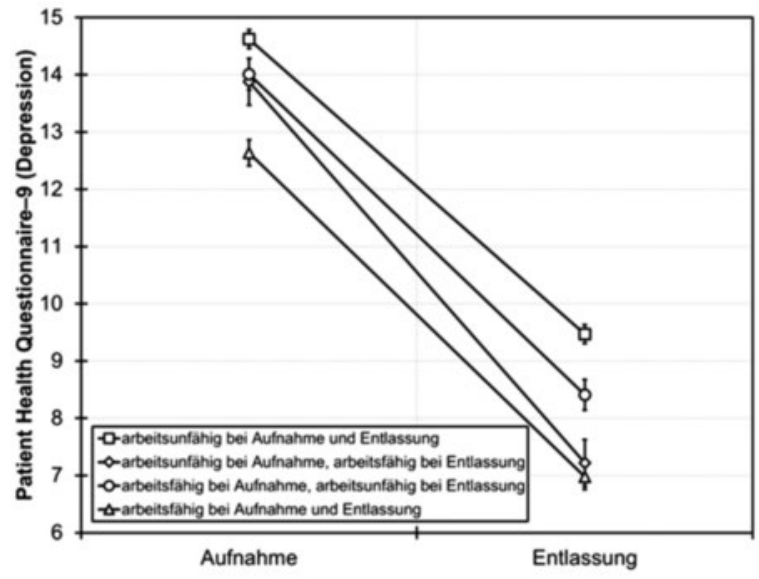

- Abb. 1 Gemittelte Summenwerte des Patient Health Questionnaire-9 bei Aufnahme und Entlassung in Abhängigkeit der Arbeitsfähigkeit vor Aufnahme und bei Entlassung. Die Fehlerbalken repräsentieren den Standardfehler des Mittelwerts.

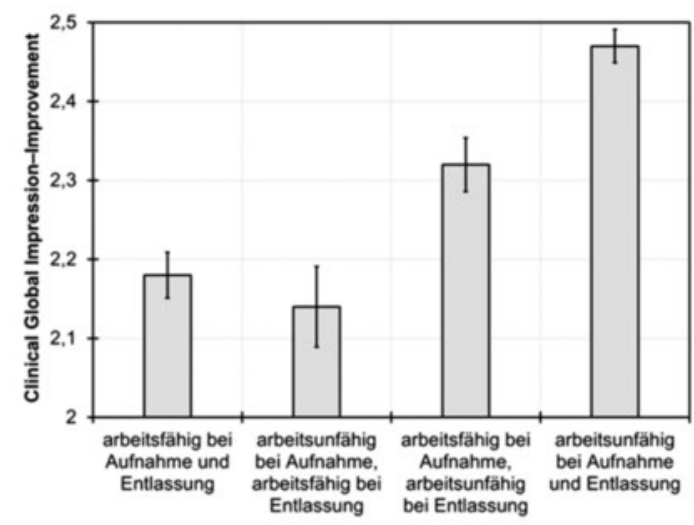

- Abb. 2 Gemittelte Werte der Clinical Global Impression-Improvement Scale in Abhängigkeit der Arbeitsfähigkeit vor Aufnahme und bei Entlassung. Die Fehlerbalken repräsentieren den Standardfehler des Mittelwerts. Man beachte, dass niedrigere Werte eine stärkere Verbesserung anzeigen.

\section{Experteneinschätzungen zum Thema Krankschreibung}

\section{Material und Methoden}

Zur Charakterisierung der Beurteilerperspektive wurden in 2 Erhebungen zum einen ärztliche und psychologische Psychotherapeuten einer psychosomatischen Klinik ( $n=73$ ) und zum anderen leitende Ärzten und Psychologen aus psychiatrischen und psychosomatischen Akut- und Rehabilitationskliniken ( $n=26)$ gebeten, einen anonymen Fragebogen zum Thema „Beurteilung der Arbeitsfähigkeit“ auszufüllen. Es ging darum, Stellung zum persönlichen Umgang mit der AU-Einschätzung zu nehmen: wurden Sie bzw. werden Ärzte und Therapeuten diesbezüg- lich angemessen ausgebildet? Ist das Thema Arbeit und Arbeitsfähigkeit Inhalt der stationären Psychotherapie? Wie sicher fühlen Sie sich in der Beurteilung der Arbeitsfähigkeit von Patienten und welche Rolle spielt dabei die Selbstbeurteilung der Patienten?

\section{Ergebnisse}

Die Einschätzungen erfahrener Ärzte und Therapeuten sowie ihrer sich in Ausbildung befindlichen Kollegen liegen eng beieinander. Darüber, dass das Thema „Beurteilung der Arbeitsfähigkeit“ in ihrer jeweiligen Ausbildung (d. h. über einige Jahrzehnte hinweg und bis heute) praktisch keine Rolle spielte ( $\triangleright$ Abb. $\mathbf{3}$, $\triangleright$ Abb. 4) und dass „Aspekte der Arbeitsfähigkeit Thema der psychotherapeutischen Arbeit sind“, besteht weitgehend Konsens. Dass die jungen Kollegen bezüglich letzterem eindeutig zustimmender antworten ( $\triangleright$ Abb. 5, $\triangleright$ Abb. $\mathbf{6})$, spiegelt vermutlich die u. a. berufsbezogenen Therapieangebote der betreffenden Klinik [21-23]. Übereinstimmend bekunden alle Befragten, dass die AU-Beurteilung schwierig ist ( $>$ Abb. 7, - Abb. 8). Mehrheitlich finden sich hier Antworten im mittleren Skalenbereich: Man habe Probleme, irgendwie macht man es trotzdem. Ähnlich verteilt sind die Antworten auf die Frage, ob Patienten letztlich selbst beurteilen, ob sie arbeitsfähig sind ( $\mathbf{A b b}$. 9, > Abb. 10). Dass diese Aussage „voll“ zutrifft konnte keiner der Ärzte/Therapeuten in Leitungsfunktion bestätigen, dass sie „nicht zutrifft" erklärten andererseits die wenigsten. Also: Zumindest haben (oder nehmen sich?) Patienten, was die Einschätzung ihrer Arbeitsfähigkeit anbelangt, oft ein gewichtiges „Mitspracherecht“.

\section{Konzeptueller Hintergrund}

Bei der Feststellung der Arbeitsunfähigkeit gelten die Grundsätze der Arbeitsunfähigkeitsrichtlinie.

\section{Arbeitsunfähigkeit}

(§ 92 Abs. 1 Satz 2 Nr. 7 SGB V, in der Fassung vom 14.11.2013). „Arbeitsunfähigkeit liegt vor, wenn Versicherte aufgrund von Krankheit ihre zuletzt vor der Arbeitsunfähigkeit ausgeübte Tätigkeit nicht mehr oder nur unter der Gefahr der Verschlimmerung der Erkrankung ausführen können. Bei der Beurteilung ist darauf zu achten, welche Bedingungen die bisherige Tätigkeit konkret geprägt haben. Arbeitsunfähigkeit liegt auch vor, wenn aufgrund eines bestimmten Krankheitszustandes, der für sich allein noch keine Arbeitsunfähigkeit bedingt, absehbar ist, dass aus der Ausübung der Tätigkeit für die Gesundheit oder die Gesundung abträgliche Folgen erwachsen, die Arbeitsunfähigkeit unmittelbar hervorrufen." (Gemeinsamer Bundesausschuss). Entscheidungsrelevant sind also die kausal aus Krankheit resultierenden Fähigkeitsstörungen und deren Auswirkung auf die individuelle Arbeitsanforderung und nicht die Krankheit selbst. 


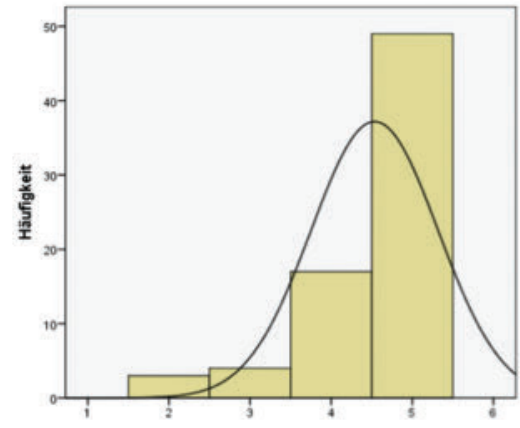

- Abb. 3 „Ärzte und Therapeuten haben im Studium gelernt, wie die Arbeitsfähigkeit von Patienten beurteilt wird“. Selbsteinschätzung von Ärzten und Psychologen ohne Leitungsfunktion: Mittelwert $=4,53$, Standardabweichung $=0,783, n=73$. Legende: $1=$ "trifft voll zu“ bis $6=$,trifft gar nicht zu“.

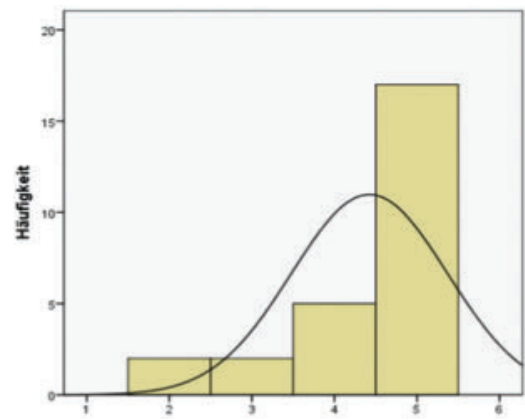

- Abb. 4 „Ärzte und Therapeuten haben im Studium gelernt, wie die Arbeitsfähigkeit von Patienten beurteilt wird“": Einschätzung von Ärzten und Psychologen mit Leitungsfunktion. Mittelwert $=4,42$, Standardabweichung $=0,945, n=26$. Legende: $1=$ „trifft voll zu“ bis $6=$,trifft gar nicht zu“.

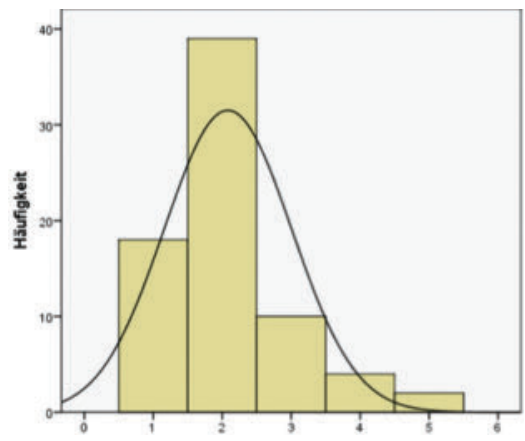

- Abb. 5 „Aspekte der Arbeitsfähigkeit sind stets auch Thema der psychotherapeutischen Arbeit": Einschätzung von Ärzten und Psychologen ohne Leitungsfunktion: Mittelwert $=2,08$, Standardabweichung $=0,924, n=73$. Legende: $0=$ keine Angabe, 1 = „trifft voll zu“ bis $6=$ „trifft gar nicht zu“.

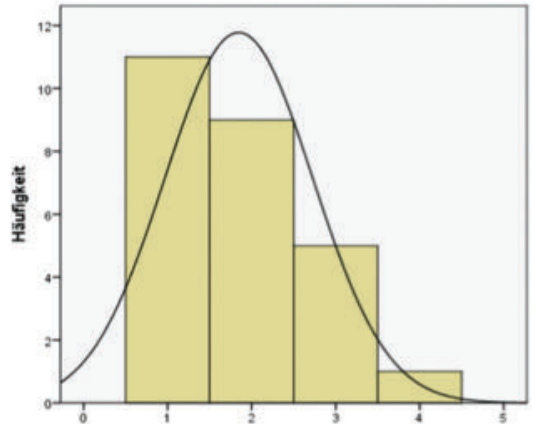

-Abb. 6 „Aspekte der Arbeitsfähigkeit sind stets auch Thema der psychotherapeutischen Arbeit": Einschätzung von Ärzten und Psychologen mit Leitungsfunktion. Mittelwert $=1,85$, Standardabweichung $=0,881, \mathrm{n}=26$. Legende: $0=$ keine Angabe, 1 = „trifft voll zu“ bis 6 = „trifft gar nicht zu“.

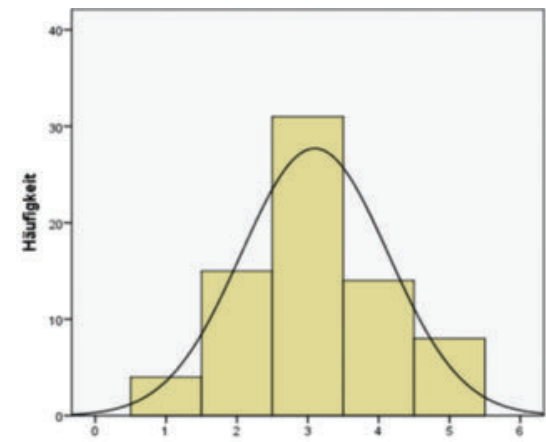

- Abb.7 „Die Arbeitsfähigkeit eines Patienten vor Entlassung zu beurteilen, ist für mich kein Problem": Selbsteinschätzung von Ärzten und Psychologen ohne Leitungsfunktion. Mittelwert $=3,1$, Standardabweichung $=1,037$, $n=72$. Legende: $0=$ keine Angabe, $1=$, trifft voll zu“ bis $6=$ „trifft gar nicht zu“.

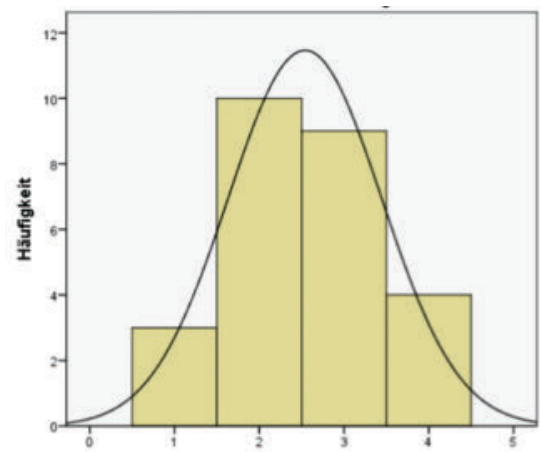

- Abb. 8 Einschätzung von Ärzten und Psychologen mit Leitungsfunktion zu der Frage „Die Arbeitsfähigkeit eines Patienten vor Entlassung zu beurteilen, ist für mich kein Problem". Mittelwert $=2,54$, Standardabweichung $=0,905, n=26$. Legende: $0=$ keine Angabe, $1=$ „trifft voll zu“ bis $6=$, trifft gar nicht zu“. 


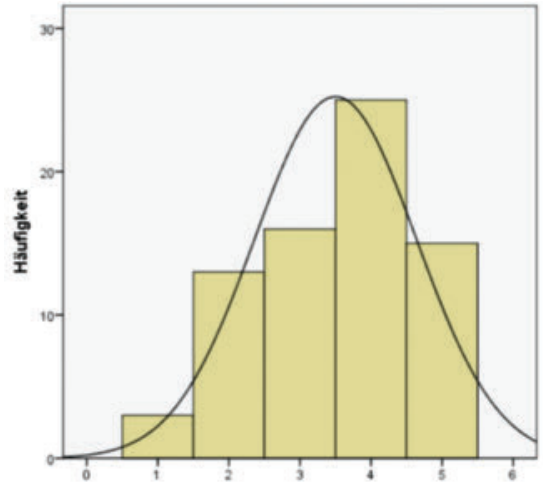

- Abb. 9 „Letztlich entscheiden Patienten selbst, ob sie arbeitsfähig sind oder nicht ...": Einschätzung von Ärzten und Psychologen ohne Leitungsfunktion. Mittelwert=3,5, Standardabweichung $=1,138, n=72$. Legende: $0=$ keine Angabe, 1 = „trifft voll zu“ bis 6 = „trifft gar nicht zu“.

\section{Diskussion}

\section{Inhaltliche bis sozialpolitische Dimensionen der Krankschreibung von psychiatrischen/psychosomatischen Patienten}

\section{Arbeitsunfähigkeit als (vermeintlich)}

\section{selbstevidenter Zustand}

Dass stationäre Psychotherapie hinsichtlich der Symptomverbesserung wirksam ist, ist hinreichend belegt [15]. Wenn Patienten trotzdem vermehrt als AU entlassen werden, wie erfolgreich war die Behandlung dann wirklich? „Die Daten sagen gar nichts, schließlich gibt es viele Gründe, warum Patienten als AU entlassen werden können", so ein Kliniker. Aus der Perspektive der Solidargemeinschaft dürfte gleichwohl kaum nachvollziehbar sein, warum deutlich gesündere Menschen vermehrt AU sein sollen und die in $\boldsymbol{A} \mathbf{A b b} \mathbf{1}$ gezeigten Zusammenhänge zwischen $\mathrm{AU}$ und Symptomverbesserung zwar tendenziell inhaltlich-logisch, aber offenkundig alles andere als trennscharf sind.

Im klinischen Alltag sind die Gründe für AU-Feststellungen offenkundig vielfältig. So "müssen“ Angestellte AU sein um eine von der Kasse finanzierte stufenweise Wiedereigliederung durchführen zu können (wobei die Indikationskriterien bzgl. einer solchen Maßnahme ihrerseits vage sind) [16]. Beamte wiederum müssen für eine solche Maßnahme teildienstfähig sein. Vielfach soll eine Entlassung als AU Patienten vor kurzfristiger Überlastung schützen und/oder sie darin unterstützen, „erst einmal gut zuhause anzukommen“, wofür Urlaub zu nehmen nicht vertretbar erscheint. Es ist zudem offenbar problematisch, Patienten gegen deren Willen als arbeitsfähig zu entlassen. Neben einer „schlechten Stimmung“ kann dies lästige Beschwerden (u. a. in Online-Portalen) zur Folge haben („Arzt hat kein Verständnis...“). Wobei zumal die letztgenannten Gründe einer AU-Beurteilung in Arztbriefen üblicherweise nicht dokumentiert werden. Dass diese im All-

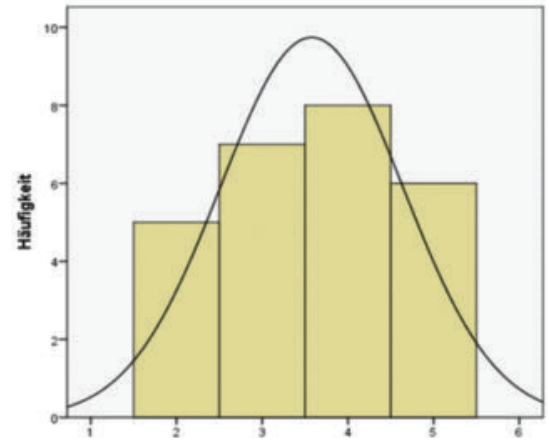

- Abb. 10 „Letztlich entscheiden Patienten selbst, ob sie arbeitsfähig sind oder nicht ...": Einschätzung von Ärzten und Psychologen mit Leitungsfunktion. Mittelwert $=3,58$, Standardabweichung $=1,065, n=26$. Legende: $1=$ „trifft voll zu“ bis $6=$, „rifft gar nicht zu“.

tag bzgl. der AU-Thematik „intermittierenden Variablen“ in der wissenschaftlichen Literatur praktisch nicht auftauchen, ist bemerkenswert [17]. Die Kosten für AU-geschriebene Patienten tragen jeweils die Arbeitgeber bzw. die Allgemeinheit [18]. Die Frage, in wieweit diese Kosten durch entsprechenden Nutzen - für alle Beteiligten - gerechtfertigt sind, war bislang kein akademisches Anliegen. Einerseits wird sozialmedizinisch davon ausgegangen, dass AU ein objektiver, fachlich- bzw. wissenschaftlich fundiert festgestellter Sachverhalt ist. Andererseits sieht es in der Praxis oft anders aus. Andreas Weber bringt es auf den Punkt [29, S. 142-144]: „Der ärztliche Alltag zeigt, dass das ... individuelle „Matching“ zwischen Anforderungen und Fähigkeiten häufig rudimentär oder gar nicht erfolgt. “ Darüber weit hinausgehend legt ein Fachbuch nahe, sich gar nicht erst für das Thema „Arbeitsfähigkeit“ zuständig zu fühlen: „Die Entscheidung über Arbeits(un)fähigkeit bzw. ggf. Erwerbsunfähigkeit liegt beim Patienten“ [17, S. 678]. Ist dies ärztlich-therapeutisch weise, eine realistische (Selbst-)Einschätzung und/oder eine Kapitulationserklärung?

\section{Arbeitsunfähigkeit und die (sozial)psychiatrische Diskussion}

Wenn, dann scheint die Frage, ob bzw. ab wann Patienten arbeits(un)fähig sind, in den Bereich der Rehabilitation zu gehören. In Therapiestudien - die über Kasuistiken hinausgehen - finden sich in der Regel keine Angaben zur beruflichen Situation der Patienten, so, als würden sich berufliche Probleme von allein lösen, wenn die jeweilige Diagnose angemessen behandelt wurde [38]. Dass von den in epidemiologischen Erhebungen z. B. als depressiv-erkrankt erkannten Menschen etwa die Hälfte nicht AU war, wird ambivalent diskutiert [6, 7]. Dass Arbeit salutogenetische Aspekte hat, u. a. was Tagesstruktur und Sozialkontakte anbelangt, ist bekannt [19], gegenüber dem Präsentismus-Problem [24-28] aber anscheinend nur schwer ab- 
zugleichen. Solange Studien zum Thema „Präsentismus“ ausschließlich auf der AU-Selbsteinschätzung der Befragten basieren, bleiben deren Ergebnisse ebenso relativ wie es Studien wären, die den volkswirtschaftlichen Schaden unangemessener AU-Schreibungen aufzeigen wollten. Je nach den jeweils zugrunde gelegten Annahmen lassen bzw. ließen sich, auf nationale Ebene hochgerechnet, jeweils unschwer Milliarden-Schadensbeträge ermitteln. Zentral ist die Diskussion um den seit Jahren statistisch offenkundigen Anstiegs von AU-Zeiten und Frühberentungen von psychisch Erkrankten [4, 5], was ebenso wie Präsentismus impliziert voraussetzt, dass es dezidierte AU-Kriterien gäbe. Im Gegensatz zur Gutachtenerstellung [30-34] wurde die AU-Feststellung wissenschaftlich bislang kaum explizit thematisiert. So wird in Studien zum Präsentismus üblicherweise die Selbst-AU-Einschätzung der Befragten als Grundlage genommen, einhergehend mit dem emotionalen Bild eines realiter Kranken, aus äußerem oder innerem Druck gezwungenermaßen auf Kosten seiner Gesundheit am Arbeitsplatz erschienenen Menschen. Ohne eingehendere Konzeptualisierung und Anwendung von reliablen und idealerweise validen AU-Kriterien ist die Interpretation dieser Daten kaum möglich. Haben sich die Arbeitsbedingungen und/oder die individuellen Maßstäbe der AU-Selbstbeurteilung (zumindest von Teilen der Bevölkerung) verändert [3]? Wenn in unserer Befragung langjährig im Beruf Tätige und deren junge Kollegen übereinstimmend bekunden, dass das AU-Thema in ihrer Ausbildung nicht hinreichend vermittelt wurde ( $>$ Abb. 3, \ Abb. 4), spiegelt dies somit angemessen eine - de facto nicht vorhandene - Forschungslage.

\section{Arbeitsunfähigkeit angesichts unterschiedlicher} Interessen

Ärzten und Therapeuten können somit nur nach bestem Wissen und Gewissen versuchen, ausgehend vom „Praxis üblichen“ und dem „Bauchgefühl“, eigene Standards zu entwickeln. Einerseits ist AU schreiben zu können eine erhebliche, weitgehende autonome Machtposition. Krankschreibungen werden kaum je überprüft, mögliche Fehlentscheidungen haben praktisch nie Konsequenzen für deren Urheber. Andererseits befinden sich Ärzte im Spannungsfeld unterschiedlicher Interessen. Patienten wollen „verstanden“ und in ihren Bedürfnissen gesehen werden, Ärzte/Therapeuten wollen ihren Patienten helfen und von diesen als verständig-kompetent erlebt werden. Arbeitgeber (bzw. „die Wirtschaft“ und damit wir alle) wollen gesunde, Leistung bringende Mitarbeiter. Konflikte mit Patienten, denen eine Krankschreibung verwehrt wir, sind oft zeitintensiv und unangenehm [35]. Bei alledem haben Ärzte/Therapeuten eigene Werte und Vorstellungen, u. a. was die Rolle und die Ethik des Arbeitgebers des Patienten anbelangt. Nicht wenige Ärzte und Therapeuten haben ihren Beruf ergriffen, weil sie nicht primär z. B. wirtschaftliche Interessen haben. Nur die wenigsten waren je selber in entsprechenden Bereichen tätig. Aus ärztlich-therapeutischer Perspektive heraus sollen Patienten lernen, auf sich zu achten und sich u. a. gegen als inadäquat erlebte Arbeitsanforderungen abzugrenzen. AU wäre dann ggf. Ausdruck persönlicher Kompetenz (,ich habe schon zu viel für die Firma geleistet...“). Dass eine Krankschreibung kurzfristig zur Entlastung, langfristig aber oft nicht zur Entschärfung von Arbeitsplatzkonflikten führt, ist relativ, dazu mitunter nur schwer vermittelbar. Letztlich müsste es darum gehen, emotional belastete Patienten durch Verbesserung ihrer Problemlösekompetenzen zu befähigen, entsprechende Konflikte - idealerweise unterstützt durch systemische Maßnahmen z. B. unter Einbeziehung des BGM - zu entschärfen, wozu „Erholung“ alleine oft nicht ausreicht. Ein systematischer Abgleich der skizzierten Perspektive findet in aller Regel nicht statt ...

\section{Kann es wissenschaftlich tragfähige AU-Kriterien} geben?

Formale Grundlage ist der zitierte Paragraph. Das Anliegen jeder Richtlinie ist, komplexe Situationen zu regeln und Eindeutigkeit (bzw. eine „Rechtsgrundlage“) herzustellen. Der Paragraph, wie bei Gesetzestexten üblich, ist aus der Metaperspektive heraus geschrieben. An zentralen Stellen verwendet er „randunscharfe“ Begriffe. Meint „Krankheit“ hier z. B., dass die Kriterien einer Diagnose im Sinne der ICD-10/-11 erfüllt sein müssen oder sollte man vom sehr viel breiter angelegten Krankheitsbegriff der WHO „Gesundheit ist ein Zustand vollkommenen körperlichen, geistigen und sozialen Wohlbefindens und nicht allein das Fehlen von Krankheit und Gebrechen “ ausgehen [37]? Angesichts dieser Unschärfen ist nicht zu erwarten, dass unterschiedliche Beurteiler angesichts eines Patienten zur gleichen AU-Einschätzung kommen. Studien dazu gibt es nicht. Zumal angesichts leichter und mittelschwerer „Fälle“, mit Blick u. a. auf Reliabilitätsuntersuchungen zu diagnostischen Kriterien [36], ist keine hohe Reliabilität zu erwarten. AU zumal bei psychischen Problemen ist offenkundig ein zu komplexes Phänomen, um eindimensional beurteilbar zu sein. Die zugrunde gelegte Diagnose bedingt dabei AU nur in Extremfällen allein. Die übrigen, im Einzelfall mehr oder weniger gewichteten Aspekte liegen - neben ggf. relevanten, testpsychologisch im Verlauf zu monitorenden Einschränkungen - auf individuellen (der des Patienten und der des Arztes/Therapeuten!) und interaktionellen Ebenen. Um diese zumindest ansatzweise berücksichtigen und beurteilen zu können, müssten AU beurteilende Ärzte angesichts eines Patienten - neben der Diagnose und deren Schweregrad:

- eingehendere Kenntnisse von der Arbeitswelt (jenseits des eigenen Faches) haben,

- ihre eigenen diesbezüglichen Einstellungen reflektieren und abstrahieren und

- die konkreten Arbeitsbedingungen eines Patienten kennen, d. h. sie dürfen nicht ausschließlich von dessen Einschätzung ausgehen [35].

In der Ausbildung kommen die ersten beiden genannten Themen (abgesehen von wenigen Arbeitsmedizineinhei- 
ten im Medizinstudium, wobei im Gegenstandskatalog - CVIII.2.6.2.4 - AU eher als kriterienfreie kommunikative Aufgabe imponiert: AU-Bescheinigung ausfüllen und das Für und Wieder mit dem Patienten abwägen) praktisch nicht vor [ 17, 21, 22]. Entsprechend müsste die ärztlich-therapeutische Ausbildung um Informations- und Reflexionseinheiten zur Arbeit in unterschiedlichen Berufsfeldern ergänzt werden. Dabei ist die hohe Dynamik aktueller Entwicklungen in der Arbeitswelt zu berücksichtigen. Diese beinhalten u. a., dass immer mehr Mitarbeiter nominell in Vorgesetztenrollen kommen, was ihnen die Möglichkeit gibt, Aufgaben zu delegieren, gleichzeitig aber auch deren Verantwortung erhöht [29, 39]. Einfache, bis heute die Literatur dominierende Beispiele, etwa wonach ein Mitarbeiter mit einem verstauchten Fuß nicht als Bote, wohl aber Rezeptionist tätig sein kann (womit der erste arbeitsunfähig, der zweite hingegen arbeitsfähig wäre), lassen sich nur bedingt auf die aktuelle (Online-)Arbeitswelt und nur bedingt auf psychische Störungsbilder übertragen. Neben der „Fähigkeit“, eine bestimmte Aufgabe auszuführen (wiederum abgesehen von Extremformen) sind realiter Aspekte der Motivation bzw. der subjektiven Konnotation der Tätigkeit mitentscheidend. Kann einem Patienten, der eine Arbeit als „unzumutbar und kränkend“ erlebt, zugemutet werden, dieses Gefühl zu überwinden [32]? In solchen Fragen hilft auch die ICF(International Classification of Functioning, Disability and Health)-Klassifikation nicht weiter, da darin im Arbeitsalltag relevante Faktoren wie „Arbeitsmotivation“ $[40,41]$ oder „Zumutbarkeit von Gratifikationskrisenkonstellationen “ [42, 43] unberücksichtigt bleiben [10, 44]. Zumal, jede längerfristige AU aufgrund psychischer Störungen lässt sich letztlich nur im Abgleich der Perspektiven des Patienten mit denen der anderen Beteiligten in seinem Arbeitsbereich - als Abwägung belastender versus salutogenetischer Faktoren - rechtfertigen. Soweit inakzeptable systemische Belastungen bestehen, sollten entsprechende Maßnahmen angestoßen werden - hier wäre die individuelle Krankschreibung nicht zielführend. Zu alledem wäre ein Dialog und Miteinander aller Beteiligten, also auch innerbetrieblichen Stellen z. B. im Rahmen des BGM, nötig.

\section{Perspektiven von der Theorie in die Praxis}

Klingt gut, aber angesichts fehlender zeitlicher Ressourcen und u. a. von Datenschutzfragen nicht umsetzbar? Was die einfachste Lösung wäre. Berufspolitisch stellt sich die Grundsatzfrage, warum Ärzte sich eine Verantwortung aufdrücken lassen, denen sie - wenn man es kritisch beleuchtet - im verlangten Modus realiter nicht gerecht werden können. Ist die damit verbundene Machtposition hinreichend attraktiv? Ein medizinisch-therapeutische, soziale und juristische Perspektiven integrierendes, objektives, realiables und valides Konstrukt „Arbeits(un)fähigkeit bei psychischen Störungen“ ist nicht vorstellbar, schon deshalb, weil die Perspektiven dynamisch sind. Wenn, dann müsste das betreffende Konzept sehr breit, also: bio-psycho-sozial, aufgestellt werden [45-47]. Statt einer AU-Bescheinigung wäre alternativ eine „Überlastungsanzeige aus medizinisch/psychotherapeutischen Gründen“ das, was ein Arzt/Therapeut (jenseits von Extremfällen und allem, was über 5 Tage hinausgeht) vertreten kann. Davon ausgehend müssten dann die im Arbeitskontext involvierten Personen (z. B. im Rahmen des BGM) Lösungen definieren und umsetzen. Mit Blick auf die Häufigkeit von Stresskonstellationen am Arbeitsplatz (die in Folge der Corona-Pandemie bedingten wirtschaftlichen Schwierigkeiten in den nächsten Jahren absehbar nicht geringer werden) erscheint das Potenzial entsprechender Modelle derart groß, dass sie - so bleibt zu hoffen - die bei deren Einführung absehbaren Widerstände überwiegen.

\section{FAZIT FÜR DIE PRAXIS}

Menschen aufgrund von psychischen Symptomen krank zu schreiben gehört zum medizinisch Alltag. Laut Sozialgesetzbuch sind dazu die „Bedingungen, die die bisherige Tätigkeit konkret geprägt haben“ mit den krankheitsbedingten Einschränkungen der betreffenden Person abzugleichen. Anhand von klinischen Daten (trotz deutlicher Symptomverbesserung werden mehr Patienten als arbeitsunfähig entlassen als aufgenommen) und Befragungen von Ärzten/Therapeuten (demnach entscheiden u.a. Patienten oft selber über ihre Krankschreibung) wird aufgezeigt, wie komplex und letztlich subjektiv das Spektrum der Krankschreibungen im Alltag zugrunde liegenden Aspekte ist. Analog zur leitlinienorientierten Behandlung läge es nahe, die Kriterien, nach denen Krankschreibungen vorgenommen werden, empirisch abzusichern. Arbeitsfähigkeit vollzieht sich stets in sozialen, sich dynamisch entwickelnden Arbeitskontexten, deren „Bedingungen“ von außen nur bedingt eingeschätzt werden können. Angesichts dessen sind autonom vom Arzt vollzogene (zumal längerfristige) Krankschreibungen oft weder gesetzeskonform noch angemessen und vielfach für die Patienten längerfristig unvorteilhaft. Alle Beteiligten einbeziehende kooperativen Modelle, selbstverständlich unter Beachtung des Datenschutzes, ist der Vorzug zu geben. 
Interessenkonflikt

Erklärung zu finanziellen Interessen

Forschungsförderung erhalten: nein; Honorar/geldwerten Vorteil für Referententätigkeit erhalten: nein; Bezahlter Berater/interner Schulungsreferent/Gehaltsempfänger: ja; Patent/Geschäftsanteile/Aktien (Autor/Partner, Ehepartner, Kinder) an Firma (Nicht-Sponsor der Veranstaltung): nein; Patent/Geschäftsanteile/Aktien (Autor/Partner, Ehepartner, Kinder) an Firma (Sponsor der Veranstaltung): nein.

Erklärung zu nicht finanziellen Interessen

Die Autoren geben an, dass kein Interessenkonflikt besteht.

\section{Korrespondenzadresse}

Prof. Dr. Dr. Andreas Hillert

Schön Klinik Roseneck, Am Roseneck 6

83209 Prien am Chiemsee, Deutschland

ahillert@schoen-klinik.de

Tel. 08051/680

\section{Literatur}

[1] Wohlers K, Hombrecher M. Entspann dich, Deutschland - TK-Stressstudie 2016. Hamburg: TK-Druckerei. https://www.tk.de/resource/blob/2026630/ 9154e4c71766c410dc859916aa798217/tk-stressstudie-2016-data. pdf

[2] Inglehart R, Welzel C. Modernization, Cultural Change and Democracy. Cambridge: Cambridge University Press, 2005

[3] Hillert A. Gebrauchsanweisung für das Leben in der Postmoderne. Stuttgart: Schattauer, 2019

[4] Bundesanstalt für Arbeitsschutz und Arbeitsmedizin. Volkswirtschaftliche Kosten durch Arbeitsunfähigkeit 2018. www. baua.de/

[5] DAK-Psychoreport 2020. https://www.dak.de/dak/bundesthemen/dak-psychoreport-2020-2335930.html\#/. Zugegriffen 20. März 2021

[6] Jacobi F, Höfler M, Strehle J, et al. Psychische Störungen in der Allgemeinbevölkerung: Studie zur Gesundheit Erwachsener in Deutschland und ihr Zusatzmodul Psychische Gesundheit (DEGS1-MH). Nervenarzt 2014; 85: 77-87. doi. org/10.1007/s00115-013-3961-y 2

[7] Jacobi F, Höfler M, Strehle J, et al. Erratum zu: Psychische Störungen in der Allgemeinbevölkerung. Studie zur Gesundheit Erwachsener in Deutschland und ihr Zusatzmodul „Psychische Gesundheit“ (DEGS1MH). Nervenarzt 2016; 87: 88-90. doi.org/10.1007/s00115-015-4458-7

[8] DGPPN, BÄK, KBV, et al. (Hrsg.). S3-Leitlinie/Nationale Versorgungsleitlinie Unipolare Depression Langfassung. 2. Aufl. 2016 Version 5. AWMF-Register-Nr.: nvl-005. https:// www.awmf.org/uploads/tx_szleitlinien/nvl-005I_S3_Unipolare_Depression_2017-05.pdf

[9] Linden M, Weidner C. Arbeitsunfähigkeit bei psychischen Störungen. Nervenarzt 2005; 76: 1421-1431

[10] Linden M. Krankheit und Behinderung. Das ICF-Modell. Nervenarzt 2015; 86: 29-35
[11] Kroenke K, Spitzer RL, Williams JBW. The PHQ-9: Validity of a brief depression severity measure. Journal of General Internal Medicine 2001; 16: 606-613. doi.org/10.1046/ j.1525-1497.2001.016009606.x

[12] Kroenke K, Spitzer RL. The PHQ-9: A new depression diagnostic and severity measure. Psychiatric Annals 2002; 32: 509-515. doi.org/10.3928/0048-5713-20020901-06

[13] Löwe B, Spitzer RL, Zipfel S, et al. PHQ-D Gesundheitsfragebogen für Patienten PHQ-D Patient Health Questionnaire 2002

[14] Busner J, Targum SD. The clinical global impressions scale: applying a research tool in clinical practice. Psychiatry 2007; 4(7): 28-37. PMID: 20526405

[15] Voderholzer U, Barton B. Langfristige Wirkung von Psychotherapie bei nichtchronischen Depressionen: Ein systematisches Review von Studien im Vergleich mit Pharmakotherapie. Verhaltenstherapie 2016; 26: 108-115. doi: 10.1159/000446674

[16] Hillert A, Weber A, Köllner V. Stufenweise Wiedereingliederung in den Arbeitsprozess: das „Hamburger-Modell“ in Psychotherapie und Psychosomatik. Psychotherapie im Dialog 2016; 2: 2-6

[17] Kröger C. Arbeitsbezogene Psychotherapie-Ansätze. In: Rief W, et al. (Hrsg.) Psychotherapie. Ein kompetenzorientiertes Lehrbuch. München: Elsevier, 2021, 669-682

[18] Trachsel M. Ethik in der Psychotherapie. In: Rief W, et al. (Hrsg.) Psychotherapie. Ein kompetenzorientiertes Lehrbuch. München: Elsevier, 2021, 743-749

[19] Strauß B. Unerwünschte Behandlungseffekte und Nebenwirkungen. In: In: Rief W, et al. (Hrsg.) Psychotherapie. Ein kompetenzorientiertes Lehrbuch. München: Elsevier, 2021, 769-775

[20] Stöckler C, Kessemeier F, Petermann F, et al. Diagnostik von Arbeitsmotivation in der psychosomatischen Rehabilitation. Identifizierung einer Rehabilitandengruppe mit ungünstigen arbeitsmotivationalen Voraussetzungen. Praxis Klinische Verhaltensmedizin und Rehabilitation 2018; 101: 5-13

[21] Hillert A, Koch S, Hedlund S. Stressbewältigung am Arbeitsplatz. Ein stationäres Gruppentherapieprogramm. Göttingen: Vandenhoeck \& Ruprecht, 2007

[22] Hillert A, Lehr D, Koch S, et al. Lehrergesundheit. AGIL - das Präventionsprogramm für Arbeit und Gesundheit im Lehrerberuf. Stuttgart: Schattauer, 2016

[23] Koch S, Lehr D, Hillert A. Burn-out und chronischer beruflicher Stress. Göttingen: Hogrefe, 2015

[24] Aronsson G, Gustafsson K, Dallner M. Sick but yet at work. An empirical study of sickness presenteeism. J Epidemiol Community Health 3000; 54(7): 502-509

[25] Johns G. Presenteeism in the workplace: a review and research agenda. J Organ Behav 2010; 31: 519-542

[26] Steinke M, Badura B. Präsentismus - Ein Review zum Stand der Forschung. Bundesanstalt für Arbeitsschutz und Arbeitsmedizin, Dortmund 2011. http://www.baua.de/de/Publikationen/Fachbeitraege/Gd60.html

[27] Lohaus D, Habermann W. Präsentismus. Heidelberg: Springer, 2018

[28] Lohaus D, Habermann W. Präsentismus: Verständnis und Einflussfaktoren. Prax Klin Verhaltensmed Rehab 2020; 109: 5-15

[29] Weber A, Peschkes L, de Boer WEL (Hrsg.). Return to Work - Arbeit für alle. Grundlagen der beruflichen Reintegration. Stuttgart: Gentner, 2015 
[30] Zeidler H. Sozialmedizinische Ergebnisse der Arbeitsunfähigkeitsbegutachtung. Offentl Gesundheitswesen 1998; 51(10): 604-607

[31] Piechowiak H, Bebensee H, Erben CM. AU-Begutachtung Wissen wir eigentlich, was wir bewirken? Gesundheitswesen 1997; 59(8-9): 483-487

[32] Hillert A. Können sozialmedizinische Gutachten zur Frage der Arbeitsfähigkeit von Menschen mit psychosomatischen Störungen „richtig“ sein? Ärztl Psychoth 2011; 6: 261-267

[33] Schneider W, Hennigsen P, Dohrenbusch R, et al. Begutachtungen bei psychischen und psychosomatischen Erkrankungen: autorisierte Leitlinien und Kommentare. Bern: Huber, 2015

[34] Goerigk S, Bühner M. Psychologische Gutachten bei klinischen Fragestellungen. In: Rief W, et al. (Hrsg.) Psychotherapie. Ein kompetenzorientiertes Lehrbuch. München: Elsevier, $2021,835-853$

[35] Hillert A, Albrecht A. Burn-out Stress Depression. Interdisziplinäre Strategien für Ärzte, Therapeuten und Coaches. München: Elsevier, 2020

[36] Spitzer RL, Forman JBW, Nee J. DSM-III field trials: Initial interrater diagnostic reliability. American Journal of Psychiatry 1979; 136: 815-817

[37] Linden H. Diagnose „Gesundheit“. Psychotherapeut 2013; 3: $249-256$

[38] Lang A, Hamann J, Brieger P. Psychosoziale und berufliche Folgen der Depression. Nervenheilkunde 2018; 9: 575-580

[39] Schmidt S, Janzon M. Der Organisations-Shift: Evolution und Transformation Ihres Unternehmens. Stuttgart: Schäffer-Poeschel, 2020

[40] Hadler NM. The illness of work incapacity. Occup Med 2016; 66(5): 346-8. doi: 10.1093

[41] Egger ST, Weniger G, Müller M. Assessing the severity of functional impairment of psychiatric disorders: equipercentile linking the mini-ICF-APP and CGI. Health Qual Life Outcomes 2019; 17: 174. doi.10.1186/s12955-019-1235-5
[42] Habermeyer B, Kaiser S, Kawohl W, et al. Rentenrelevante Arbeitsunfähigkeit und Mini-ICF-APP. Neuropsychiatr 2017; 31(4): 182-186. doi: 10.1007/s40211-017-0246-x

[43] Siegrist ]. The effort-reward imbalance model. Occupational Medicine: State of the Art Reviews 2000; 15(1): 83-87

[44] Siegrist J, Wahrendorf M (Hrsg.). Work stress and health in a globalized economy. The model of efford-reward imbalance. Heidelberg: Springer, 2016

[45] Väänänen A, Pahkin K, Huuhtanen P, et al. Are intrinsic motivational factors of work associated with functional incapacity similarly regardless of the country? J Epidemiol Community Health 2005; 59(10): 858-63. doi: 10.1136/ jech.2004.030106

[46] van der Molen HF, de Vries S, Sluiter JK. Occupational Diseases among Workers in Lower and Higher Socioeconomic Positions. Int J Environ Res Public Health 2018; 15(12): 2849. doi: 10.3390/ijerph15122849

[47] Seglem KB, Torvik FA, Røysamb E, et al. A Life Course Study of Genetic and Environmental Influences on Work Incapacity. Twin Res Hum Genet 2020; 23(1): 16-22. doi: 10.1017/ thg.2019.116

\section{Bibliografie}

Nervenheilkunde 2021; 40: 884-892

DOI 10.1055/a-1500-2596

ISSN $0722-1541$

(c) 2021. Thieme. All rights reserved. Georg Thieme Verlag KG, Rüdigerstraße 14, 70469 Stuttgart, Germany 


\section{Punkte sammeln auf CM/F.thieme.de}

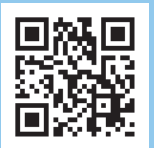

Diese Fortbildungseinheit ist bis zu 12 Monate online für die Teilnahme verfügbar.

Den genauen Einsendeschluss finden Sie beim Modul auf https://cme.thieme.de/CXHHR2X.

Sollten Sie Fragen zur Online-Teilnahme haben, finden Sie unter https://cme.thieme.de/hilfe

eine ausführliche Anleitung. Wir wünschen viel Erfolg beim Beantworten

der Fragen!

Unter https://eref.thieme.de/CXHHR2X oder über den QR-Code kommen Sie

direkt zum Artikel.

VNR 2760512021160211201

\section{Frage 1}

Welche Aussage trifft nicht zu?

A Die Selbsteinschätzung der Patienten ist ein wichtiger Faktor bei der Arbeitsunfähigkeitsfeststellung.

B Die Ablehnung eines Arztes, eine Arbeitsunfähigkeitsbescheinigung auszustellen, kann zu Konflikten mit den betreffenden Patienten führen.

C Im Medizinstudium und in der medizinischen Weiterbildung werden keine eindeutigen Kriterien für die Feststellung einer Arbeitsunfähigkeit vermittelt.

D Betriebsärzte können wichtige Ansprechpartner bei der Beurteilung einer Arbeitsunfähigkeit sein, da sie über Arbeitsplatzkenntnisse verfügen.

E Ärzte in Leitungsfunktionen haben kaum Schwierigkeiten, die Arbeitsfähigkeit von Patienten zu beurteilen.

\section{Frage 2}

Voraussetzung einer Arbeitsunfähigkeit ist definitionsgemäß:

A Eine schnelle Aussicht auf prognostische Besserung der Erkrankung durch die Arbeitsunfähigkeitsbescheinigung.

B Eine Gefahr einer Verschlimmerung der Erkrankung bei weiterer Ausübung der zuletzt ausgeübten Tätigkeit.

C Eine Einschränkung nach der International Classification of Functioning, Disability and Health (ICF).

D Das Vorliegen eines ungekündigten Arbeitsverhältnisses.

E Ein ursächlicher Zusammenhang zwischen Tätigkeit und Erkrankung.

\section{Frage 3}

BGM steht als Abkürzung für:
A Betroffeneninitiierte Gesundheitsmaßnahme
B Bundesgesundheitsministerium
C Betriebliches Gesundheitsmanagement
D Berufsgenossenschaftlicher Maßnahmenkatalog
E Behavioral guided management

\section{Frage 4}

Welche Antwort ist richtig? Für die Bescheinigung von Arbeitsunfähigkeit bei stufenweisen Wiedereingliederungsverfahren ...

A muss zwischen Angestellten und Beamten unterschieden werden.

B darf keine gesundheitliche Einschränkung der Betroffenen mehr vorliegen.

C ist eine vorherige stattgefundene stationäre Behandlung Voraussetzung.

D ist gleichzeitig eine Überlastungsanzeige aus medizinisch/ psychologischen Gründen zu stellen.

E müssen eingehende Kenntnisse der Arbeitswelt vorliegen.

\section{Frage 5}

Die besten Ergebnisse in der Clinical Global Impression-Improvement Scale (CGI) zeigen sich ...

A bei Therapiebeginn und Entlassung arbeitsfähiger Patienten.

$B$ bei Therapiebeginn und Entlassung arbeitsunfähiger Patienten.

C bei Therapiebeginn arbeitsunfähiger Patienten, die bei Entlassung arbeitsfähig sind.

D bei Therapiebeginn arbeitsfähiger und bei Entlassung arbeitsunfähiger Patienten.

$\mathrm{E}$ in allen Gruppen gleich verteilt.

\footnotetext{
- Weitere Fragen auf der folgenden Seite ...
} 
Punkte sammeln auf CME.thieme.de

Fortsetzung ...

\section{Frage 6}

Welche Aussage trifft zu?

A Krankenkassenstatistiken belegen eine seit Jahren zunehmende Häufigkeit von Krankschreibungen aufgrund psychischer Diagnosen, ohne dass psychische Erkrankungen in der erwachsenen Bevölkerung insgesamt zugenommen haben.

B Krankenkassenstatistiken belegen eine seit Jahren gleichbleibende Häufigkeit von Krankschreibungen aufgrund psychischer Diagnosen, da psychische Erkrankungen in der erwachsenen Bevölkerung nicht zugenommen haben.

C Krankenkassenstatistiken belegen eine seit Jahren abnehmende Häufigkeit von Krankschreibungen aufgrund psychischer Diagnosen, da psychische Erkrankungen nur bei Kindern und Jugendlichen zugenommen haben.

D Krankenkassenstatistiken belegen eine seit Jahren zunehmende Häufigkeit von Krankschreibungen aufgrund psychischer Diagnosen, da psychische Erkrankungen in der erwachsenen Bevölkerung stark zugenommen haben.

E Krankenkassenstatistiken zur Häufigkeit von Krankschreibungen aufgrund psychischer Diagnosen sind nicht verfügbar.

\section{Frage 7}

Welche Aussage trifft zu?

A Es liegen keine Daten zur Arbeitsfähigkeit nach stationär psychosomatischer Behandlung vor.

B Daten zur Arbeitsfähigkeit nach stationärer psychosomatischer Behandlung zeigen, dass diese bei den meisten Patienten wieder gegeben war.

C Entsprechend dem Ergebnis einer empirischen Erhebung gibt es viele Patienten, die trotz deutlicher Symptomverbesserung als arbeitsunfähig entlassen werden.

D Es gibt keinen Zusammenhang zwischen psychischen Erkrankungen und Arbeitsfähigkeit und demnach liegen hierzu auch keine Ergebnisse empirischer Erhebungen vor.

E Bei guten Behandlungsergebnissen kann automatisch auch davon ausgegangen werden, dass der betroffene Patient (wieder) arbeitsfähig ist.

\section{Frage 8}

Die Arbeitsfähigkeit bzw. Arbeitsunfähigkeit einzuschätzen ist eine im Alltag häufige ärztliche Aufgabe. Welche Aussage hierzu trifft nicht zu?

A Wenn eine Krankschreibung verweigert wird, kann dies das Verhältnis zwischen Arzt und Patient belasten.

B Im Rahmen der ärztlichen und therapeutischen Behandlung ist es wichtig, arbeitsbezogene Probleme von Patienten offen anzusprechen.

C Da letztlich Patienten selbst bestimmen, ob sie arbeitsfähig sind oder nicht, sollte dieses emotionale Thema in der ambulanten und stationären Therapie - abgesehen von Rehabilitationsbehandlungen - ausgespart werden.

D Um Arbeitsunfähigkeit angemessen beurteilen zu können, ist es - auch laut Sozialgesetzbuch - wichtig, über die Aussagen eines Patienten hinaus über dessen reale Arbeitsbedingungen informiert zu sein.

E Empirische Forschung zur Frage, wie objektive, reliable und letztlich valide Kriterien von Krankschreibungen von unter psychischen Störungen leidenden Patienten aussehen könnten, gibt es bislang praktisch nicht.

\section{Frage 9}

Psychotherapie fokussiert traditionell auf die Bearbeitung/Bewältigung biografischer Belastungen und/oder die jeweilige Symptomatik eines Patienten. Wie sollte mit arbeitsbezogenen Problemen von Patienten umgegangen werden? Welche Aussage hierzu trifft zu?

A Wenn Patienten über aktuelle arbeitsbezogene Themen berichten, dann wollen sie damit in der Regel die Thematisierung belastender Aspekte ihrer frühen Biografie vermeiden.

B Parallel zu einer leitliniengerechten Behandlung der jeweiligen Symptomatik bzw. Diagnose reduzieren sich die arbeitsbezogenen Probleme der Patienten.

C Die offene Thematisierung eventueller Arbeitsplatzkonflikte ist für Patienten derart belastend, dass dies nach Möglichkeit vermieden werden sollte.

D Eine offensive Thematisierung arbeitsplatzbezogener Probleme von Patienten ist notwendig, um die Therapie angemessen planen zu können.

E Wenn Patienten berufsbezogene Probleme haben, müssen sie an einen Coach überwiesen werden. 


\section{Punkte sammeln auf CM/E.thieme.de}

Fortsetzung $\ldots$

\section{Frage 10}

Wie wird Gesundheit von der WHO definiert?

A Gesundheit ist ein virtueller Idealzustand, der sich letztlich nicht definieren lässt und zu dem entsprechend auch keine Definitionen vorliegen.

B Gesundheit bezieht sich ausschließlich auf das körperliche Wohlbefinden eines Menschen.

C Gesundheit ist ein Zustand vollkommenen körperlichen, geistigen und sozialen Wohlbefindens und nicht allein das Fehlen von Krankheit und Gebrechen.

D Gesundheit besteht immer dann, wenn ein Mensch nicht krank ist.

E Für den Gesundheitsbegriff sind geistige und soziale Faktoren unerheblich. 